\title{
EDITORIALS
}

\section{Health Information Technology and Disparities in Quality of Care}

\author{
Thomas D. Sequist, MD, MPH

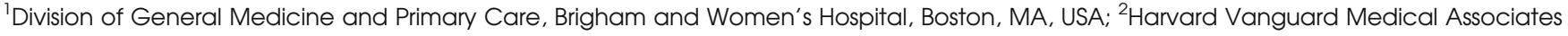 \\ and Atrius Health, Boston, MA, USA; ${ }^{3}$ Department of Health Care Policy, Harvard Medical School, Boston, MA, USA.
}

$\mathrm{J}$ Gen Intern Med 26(10):1084-5

DOI: $10.1007 / \mathrm{s} 11606-011-1812-8$

(c) Society of General Internal Medicine 2011

$\mathrm{T}$ he persistence of disparities in health care is well documented. The 2010 Agency for Healthcare Research and Quality National Healthcare Disparities Report shows that racial and ethnic disparities in quality and access are not improving, and in some cases may be worsening. ${ }^{1}$ These data create urgency for health care delivery system and health policy leaders to develop solutions to improve the health of minority populations.

Is health information technology the solution? Multiple key functionalities have been suggested, including the concept of exchanging health information among multiple providers and their patients, use of clinical decision support and computerized physician order entry, and the ability to better engage in population management via electronic disease registries and clinical dashboard reports. There is growing evidence available to quantify these benefits, although currently much of the evidence comes from a few large, integrated health care organizations. ${ }^{2,3}$

Despite considerable potential, health information technology will not reduce health care disparities if the technology remains out of reach to safety net providers and the patients they serve. Some studies have shown reduced availability of electronic health records for providers caring for underserved populations. Other studies have demonstrated that racial and ethnic minorities are less likely to engage actively in the use of specific health information technology tools such as personal health records or patient portals. ${ }^{4}$

In this issue of JGIM, two studies contribute to the growing evidence base around disparities in the use of health information technology. ${ }^{5,6}$ Goel et al. studied a single group practice affiliated with an academic medical center, and analyzed use of a patient portal that is directly integrated within a commercial electronic health record. In this setting, providers offer enrollment to their patients, and these offers were tracked via the placement of electronic orders. Among those offered enrollment, black and Hispanic patients were up to $67 \%$ less likely than whites to enroll in the patient portal. However, there were no racial or ethnic differences in rates of using the patient portal for medical advice or prescription refills among those patients that successfully enrolled.

In the study by Ancker et al., the same electronic health record and patient portal were analyzed; however, the setting

Published online August 2, 2011 was quite different as it involved a network of federally qualified health centers. These investigators found racial disparities across the entire spectrum of engaging patients in effective use of the portal, with lower rates of offering enrollment among blacks compared to whites, as well as lower rates of subsequent registration and usage of the patient portal. ${ }^{6}$

These studies raise several important issues as we consider the role of health information technology in addressing racial disparities in quality of care. It is vital to develop an evidence base regarding the impact of differential access to health information technology on quality of care and outcomes for minority patients. Most studies have focused on measuring access to health information technology, assuming that decreased access will eventually lead to persistence or worsening of disparities in quality of care. However, it is not clear whether minority patients benefit from such technologies to the same extent as white patients.

To better conceptualize whether racial differences in access to health information technology may propagate or eliminate racial disparities in quality of care, it is useful to consider the target users. Targeted users include health care providers, their patients, or a combination of both. Perhaps the most important health information technology targeting providers is the electronic health record. This tool can deliver real-time, patient-specific clinical decision support and also supply data to populate and maintain electronic disease registries. The benefits of these tools have been demonstrated, ${ }^{2}$ and while race-stratified analyses are lacking, it is likely that there would be beneficial effects of extending these tools to providers caring for minority populations. Health care reform and meaningful use requirements may serve as a driving force for adoption in these communities. ${ }^{7}$ The requirement to collect race, ethnicity, and preferred language for patients presents a key opportunity to mitigate health disparities. Systematic collection of these data will facilitate measurement of racial and ethnic disparities at a local level, fostering engagement among providers and spurring the development of quality improvement programs to better address the needs of minority patients. ${ }^{8}$

It is important to consider scenarios in which the adoption of electronic health records may actually increase health disparities. First, low adoption rates among providers caring for minority populations can lead to increased disparities if the benefits detailed above are more available to providers caring for white patients. ${ }^{9}$ However, even after equal access to electronic medical records is achieved, it is entirely possible that gains achieved by white patients as a result of electronic medical record use by their providers may not be realized by minority patients, creating a widening gap in health outcomes. For example, electronic health records are often used as a platform for population management and patient outreach via 
the creation of electronic disease registries. If these activities occur without attention to cultural appropriateness, proper reading level, or preferred language, the benefits may only accrue to more educated patients or those who are proficient in English.

Health information technologies targeting patients include personal health records, patient portals, and web-based and social media technology. Personal health records provide an avenue for patients to directly access and transfer their health information, which may ultimately lead to better coordination of care and health care engagement. Integrated patient portals such as the one analyzed by Goel et al. and Ancker et al. also provide a mechanism for providers to reach out directly to patients to promote positive health behaviors. ${ }^{10}$ Given the potential for improving health care quality via use of patient portals, it is important to understand why minority patients may not use this technology. First, these patients may not be fully aware of the existence of the tool if providers preferentially offer enrollment to white patients, potentially due to a perception of who might be more willing or able to use patient portals. This phenomenon appears to be a very real possibility based on the analyses provided by Ancker et al., with whites 60\% more likely than blacks to receive an access code for the patient portal.

However, both studies in this issue of JGIM demonstrate that even among patients who are offered enrollment, white patients are more likely to register as users. This may be due to a lack of perceived benefit among minorities, potentially related to the activities facilitated by the patient portal or the interface provided by the portal. Emerging data suggest that minority individuals have equal access to the internet compared to whites but preferentially access the internet via mobile devices including smartphones. For these patients, portals designed for desktop use may hold little allure. ${ }^{4}$

This contrast highlights a potential mechanism where a health information technology tool might preferentially benefit white patients, even when access is perceived as being equal. Health system leaders and innovators may need to better understand the perceived needs of minority patients, and how these needs can be addressed using tools that take advantage of mobile technology. This may involve using online social media as a mechanism of patient education and shared learning and support, as well as use of text messaging to deliver succinct health promotion messages from the provider to the patient.

Telemedicine represents an excellent example of a health information technology tool that can target both providers and patients. Telemedicine may hold the most promise for addressing health care disparities that are specifically related to lack of access to specialty care and other resources typically available only at large, tertiary medical centers. Telephone or video conferencing can be used to support regular communication between primary care providers working in underserved settings and specialist physicians working at academic medical centers, with the goal of expanding the capability of these primary care providers to provide complex care. ${ }^{11}$
Patients may also receive direct consultation, evaluation, and treatment via telemedicine to expand access and quality of care. The Joslin Vision Network program provides one such example where digital screening for diabetic retinopathy can take place in the physical absence of specially trained eye care professionals. Retinal images are interpreted at referral centers, and subsequent treatment recommendations provided by these centers. This program has increased both screening and treatment rates for diabetic retinopathy in the Indian Health Service, which is providing care for American Indian/ Alaska Natives in resource-poor settings, often with limited access to ophthalmologic services. ${ }^{12}$

In summary, health information technology holds great promise for improving health care delivery for all Americans. However, as we implement these tools, we need to pay attention to how such programs will affect our most vulnerable patients.

Corresponding Author: Thomas D. Sequist, MD, MPH; 1620 Tremont Street, Boston, MA 02120, USA (e-mail: tsequist@partners. org).

\section{REFERENCES}

1. Agency for Healthcare Research and Quality. National Health Care Disparities Report. Rockville, MD 2010.

2. Buntin MB, Burke MF, Hoaglin MC, Blumenthal D. The benefits of health information technology: a review of the recent literature shows predominantly positive results. Health Aff (Millwood). 2011;30:464-471.

3. Chaudhry B, Wang $\mathbf{J}$, Wu S, et al. Systematic review: impact of health information technology on quality, efficiency, and costs of medical care. Ann Intern Med. 2006; 144:742-752.

4. Gibbons MC. Use of health information technology among racial and ethnic underserved communities. Perspectives in health information management. 2011:1-13.

5. Goel MS, Brown TL, Williams A, Hasnian-Wynia R, Thompson JA, Baker DW. Disparities in enrollment and use of an electronic patient portal. J Gen Intern Med. 2011; doi:10.1007/s11606-011-1728-3.

6. Ancker JS, Barron Y, Rockoff ML, et al. Use of an electronic patient portal among disadvantaged populations. J Gen Intern Med. 2011; doi: 10.1007/s11606-011-1749-y.

7. Blumenthal D, Tavenner M. The "meaningful use" regulation for electronic health records. N Engl J Med. 2010;363:501-504.

8. Sequist TD, Fitzmaurice GM, Marshall R, et al. Cultural competency training and performance reports to improve diabetes care for black patients: a cluster randomized, controlled trial. Ann Intern Med. 2010;152:40-46.

9. Hing E, Burt CW. Are there patient disparities when electronic health records are adopted? J Health Care Poor Underserved. 2009;20:473-488.

10. Sequist TD, Zaslavsky AM, Colditz GA, Ayanian JZ. Electronic patient messages to promote colorectal cancer screening: a randomized controlled trial. Arch Intern Med. 2011;171:636-641.

11. Arora S, Thornton K, Murata G, et al. Outcomes of treatment for hepatitis C virus infection by primary care providers. N Engl J Med. 2011;364:2199-2207.

12. Wilson C, Horton M, Cavallerano J, Aiello LM. Addition of primary care-based retinal imaging technology to an existing eye care professional referral program increased the rate of surveillance and treatment of diabetic retinopathy. Diabetes Care. 2005;28:318-322. 